\title{
Factores asociados al grado de satisfacción del usuario del consultorio externo de gastroenterología del Hospital Central de la Fuerza Aérea del Perú. Mayo, 2014*
}

\author{
Associated Factors to the satisfaction degree of the user of the external gastroenterology clinic of the Central \\ Hospital of the Peruvian Air Force. May, 2014
}

Fatores associados ao grau de satisfação do usuário do consultório de gastrenterologia do Hospital Central da Força Aérea peruana. Maio, 2014

\author{
Angello Rafael La Torre Mantilla ${ }^{\text {a }}$ \\ Universidad de San Martín de Porres, Perú \\ angellolatorre@hotmail.com \\ ORCID: http://orcid.org/0000-0002-7642-3101 \\ Alfredo Enrique Oyola García \\ Natural and Social Sciences Research, Perú \\ ORCID: http://orcid.org/0000-0002-4560-7776 \\ Melisa Pamela Quispe Ilanzo \\ Natural and Social Sciences Research, Perú \\ ORCID: http://orcid.org/0000-0003-3695-591X
}

DOI: https://doi.org/10.11144/Javeriana.rgps17-34.fasu

Fecha de recepción: 31 Marzo 2017

Fecha de aceptación: 06 Octubre 2017

\section{Resumen:}

Se desarrolló un estudio observacional, descriptivo y transversal para determinar los factores asociados al grado de satisfacción del usuario del consultorio externo de gastroenterología del Hospital Central de la Fuerza Aérea del Perú, debido a que la insatisfacción del paciente se asocia con el nivel de excelencia profesional, uso eficiente de los recursos y mínimo riesgo para el paciente. La muestra incluyó 221 pacientes. Se halló que 21.3\% de pacientes estaban satisfechos con la atención, 26,7\% en el componente fiabilidad, $31.2 \%$ en el componente responsabilidad, $48 \%$ en el componente seguridad, $38.9 \%$ en el componente empatía y $32.6 \%$ en el componente tangibles. La insatisfacción se observó en 75 (70.8\%) de los varones, 99 (86.1\%) de las mujeres $(\mathrm{p}=0.005)$, en $65(70.7 \%)$ de los beneficiarios directos y 109 (84.5\%) de los familiares ( $\mathrm{p}=0.013)$. Asimismo, conforme aumentaba el tiempo de espera para la atención, disminuía la satisfacción del paciente $(\mathrm{r}=-0.316 ; \mathrm{p}=0.000)$.

Palabras clave: satisfacción del paciente, calidad de la atención de salud, hospitales militares, atención ambulatoria, servicios de salud, gastroenterología.

\begin{abstract}
:
An observational, descriptive and cross-sectional study was performed to determine the factors associated with the degree of user satisfaction at the outpatient Gastroenterology Service in the Air Force's Central Hospital from Peru, because the patient's insatisfaction is associated to the level of professional excellence, the efficient use of resources and very low risk for the patient. The sample consisted of 221 patients. It was found that $21.3 \%$ patients were satisfied with the care provided, $26.7 \%$ in the reliability component, $31.2 \%$ in the liability component, $48 \%$ in the security component, $38.9 \%$ in the empathy component and $32.6 \%$ in the tangible component. Insatisfaction was observed in 75 (70.8\%) of males, 99 (86.1\%) of females ( $\mathrm{p}=0.005), 65$ (70.7\%) of direct beneficiaries and $109(84.5 \%)$ of the family members $(\mathrm{p}=0.013)$. Also, as the waiting time for care increased patient's satisfaction decreased $(\mathrm{r}=-0.316, \mathrm{p}=0.000)$.
\end{abstract}

Keywords: patient satisfaction, quality of healthcare, military hospitals, ambulatory care, health services, gastroenterology.

\section{Resumo:}

Desenvolveu-se um estudo observacional, descritivo e transversal para determinar os fatores associados ao grau de satisfação do utente da clínica médica ambulatória de gastrenterologia do Hospital Central da Força Aérea de Peru, devido a que a insatisfação do paciente é associada com o nível de excelência profissional, uso eficiente dos recursos e mínimo risco para o paciente. A amostra incluiu 221 pacientes. Achou-se que $21.3 \%$ de pacientes estavam satisfeitos com o atendimento, 26,7\% com o componente fiabilidade, $31.2 \%$ no componente responsabilidade, $48 \%$ no componente segurança, $38.9 \%$ no componente empatia e $32.6 \%$ com

\footnotetext{
a Autor de correspondencia. Correo electrónico: angellolatorre@hotmail.com
} 
no componente tangíveis. A insatisfação observou-se em 75 (70.8\%) dos homens, 99 (86.1\%) das mulheres (p=0.005), 65 (70.7\%) dos beneficiários direitos e $109(84.5 \%)$ dos parentes $(\mathrm{p}=0.013)$. Além disso, à medida que aumentava o tempo de espera para o atendimento, diminuía a satisfação do paciente $(r=-0.316 ; \mathrm{p}=0.000)$.

Palavras-chave: satisfação do paciente, qualidade da atenção de saúde, hospitais militares, atendimento ambulatório, serviços de saúde, gastroenterologia.

\section{Introducción}

La calidad en salud es definida como un alto nivel de excelencia profesional y el uso eficiente de los recursos con un mínimo de riesgos para el paciente, a fin de lograr un alto grado de satisfacción en este y un impacto final positivo en su salud $[1,2,3,4]$. Las dimensiones que permiten su medición en los servicios de salud están referidas a la calidad científico- técnica, a la satisfacción de los usuarios y a la accesibilidad a ellos $[1,2,5,6,7,8,9]$.

El grado de satisfacción del usuario se mide mediante la diferencia existente entre la expectativa y su percepción. Esta será influenciada por factores individuales, familiares, sociales y relacionados con el servicio de salud $[8,10,11,12,13]$. Por tanto, la mala calidad de la atención se asocia con insatisfacción del paciente, que se refleja en quejas, reclamos y denuncias en los medios de comunicación, impacto en la sociedad, desprestigio de las instituciones y, en algunos casos, en procesos judiciales $[1,14,15,16]$. De esta forma, la opinión de los usuarios de los servicios de salud es importante en cuanto a futuros replanteamientos de la planificación de servicio y en lo referente a la participación de la comunidad en la orientación y ordenación de dichos servicios $[10,17,18,19,20]$.

Según algunos estudios, los niveles de satisfacción de usuario en atenciones ambulatorias oscilan entre 60\% y $82 \%$, según el público estudiado, servicio o establecimiento proveedor de la atención, motivo de consulta, momento de entrevista y método utilizado $[10,21,22,23,24,25]$. En un estudio colombiano, Cabrera-Arana et al. [26] observaron que el trato humanizado recibido de los funcionarios asistenciales fue la característica de la calidad de la atención mejor valorada. En el Perú, Chávez et al. [27] señalan que la satisfacción del paciente se asocia a una buena información acerca de su enfermedad por parte del médico. Por su parte, Ortiz et al. [28] hallaron que la insatisfacción de la persona se relaciona con las omisiones en el proceso de consulta, con el maltrato por parte del médico y con la percepción de mala calidad de atención, mientras que en el estudio de Andía et al. [23] los usuarios estaban menos satisfechos con el tiempo de espera para la consulta y para tópico, obtener cita, calidad de los medicamentos, tiempo que demoran los resultados y percepción del monto que aportan por su seguro.

En el marco del Sistema de Gestión de la Calidad, el Ministerio de Salud ha establecido estándares, indicadores e instrumentos técnicos que permiten levantar información confiable de la calidad de los servicios de salud, en los que se incluye la satisfacción del usuario [29]. Estas son guías rectoras en los establecimientos de salud del sector. Uno de estos establecimientos es el Hospital Central de la Fuerza Aérea de nivel III, que forma parte del Ministerio de Defensa del Perú y, dentro de este, el Servicio de Gastroenterología es el encargado de brindar atención integral a los pacientes con enfermedades del aparato digestivo $[5,6]$.

En este contexto, debido a que el consultorio externo de gastroenterología tiene elevada demanda de atención entre los servicios que oferta el Hospital Central de la Fuerza Aérea del Perú, y al existir pocos estudios publicados que evalúen el comportamiento y el grado de satisfacción del paciente gastroenterológico atendido en consultorios externos de hospitales del país y a nivel internacional $[5,6,10]$, nos propusimos desarrollar la presente investigación con el objetivo de determinar los factores asociados al grado de satisfacción del usuario del consultorio externo de gastroenterología del Hospital Central de la Fuerza Aérea del Perú. 


\section{Método}

Se desarrolló un estudio observacional, descriptivo de corte transversal. La población estuvo constituida por los pacientes atendidos en el consultorio externo del Servicio de Gastroenterología del Hospital Central de la Fuerza Aérea del Perú. Durante el año 2013, este número llegó a 2000 pacientes. La muestra —221 pacientes - se halló con la siguiente fórmula: $n=Z \alpha 2 p q N /(E 2 N+Z \alpha 2 p q)$, donde: $Z \alpha=$ coeficiente de confiabilidad $(95 \%)=1.96 ; \mathrm{p}=$ proporción estimada del problema en la población $=0.2 ; \mathrm{q}=(1-\mathrm{p})=0.8 ; \mathrm{E}$ = error absoluto $(5 \%)=0.05 ; \mathrm{N}=$ población $=2000$ pacientes; $y$ fue seleccionada en el mes de mayo del 2014 mediante muestreo aleatorio simple. Se incluyeron; hombres/mujeres, militares (beneficiario directo) y civiles (familiar de militar), nuevos (primera consulta) o continuadores (acude por segunda consulta o posterior) atendidos en el consultorio de gastroenterología del Hospital Central de la Fuerza Aérea del Perú, con rango de edad (16 a 55 años) y que aceptaron participar en el estudio mediante consentimiento informado. Se excluyó a los pacientes que no estaban interesados en participar en la investigación.

Se usó el cuestionario Servqual modificado, propuesto en la Guía técnica para la evaluación de la satisfacción del usuario externo en los establecimientos de salud y servicios médicos de apoyo, propuesta por el Ministerio de Salud del Perú [30], que contiene veintidós pares de preguntas. Las modificaciones de este cuestionario fueron desarrolladas y validadas por Cabello y Chirinos [31], tomando como base el cuestionario original con cinco dimensiones de la calidad, distribuidas en veintidós preguntas de percepciones y expectativas, al que se incluyeron nuevos constructos: las características de calidad desde la percepción de los usuarios externos de consulta externa y las principales actividades del proceso de atención. Para la calificación de las expectativas y la percepción se usó una escala numérica del 1 al 7 , considerando 1 la más baja y 7 la más alta. El instructivo para solicitar las expectativas decía:

"Estimado usuario, califique entre 1 a 7 la importancia que usted le otorga a cada una de las siguientes preguntas relacionadas con la atención que usted espera recibir en el consultorio externo de Gastroenterología del Hospital Central de la Fuerza Aérea del Perú. Considere a 1 como la menor califi cación y 7 como la mayor calificación."

Y para la percepción:

"Estimado usuario, califique entre 1 a 7 a cada una de las siguientes preguntas relacionadas con la atención que usted ha recibido en el consultorio externo de Gastroenterología del Hospital Central de la Fuerza Aérea del Perú. Considere a 1 como la menor calificación y 7 como la mayor calificación.”

Las expectativas se solicitaron haciendo mención del establecimiento y del servicio donde sería atendido el usuario, y no sobre un referente hipotético donde se brinda "excelente calidad de atención", como recomienda la metodología original Servqual. La validación del constructo mostró una medida de adecuación de KMO de 0.975. La prueba de esfericidad de Bartlett para ambas mostró un valor signifi cativo ( $\mathrm{p}<0.001)$. Los cinco componentes o dimensiones de la calidad: fiabilidad $(\mathrm{F})$, capacidad de respuesta (CR), seguridad (S), empatía (E) y aspectos tangibles (T) explicaron el 88.9 de la varianza de las veintidós preguntas. Su alfa de Cronbach es igual 0.98 [31]. Esto demuestra características psicométricas de validez, alta confiabilidad y aplicabilidad que la hacen recomendable para su uso en la consulta externa.

Este cuestionario fue entregado a cada paciente que llegó a la consulta externa del servicio de gastroenterología del hospital durante siete días y su desarrollo tuvo una duración promedio de diez a quince minutos. Al finalizar la aplicación de la encuesta se realizó el control de calidad previo a la digitación, verificando que todas las preguntas fueron respondidas.

Se aplicaron las siguientes técnicas de procesamiento de datos: a) ordenamiento y clasificación, para tratar la información cualitativa y cuantitativa en forma ordenada, de modo de interpretarla y sacarle el máximo provecho; b) registro manual, para digitar la información de las diferentes fuentes; c) Proceso computarizado con el software IBM SPSS (Statistical Package of the Social Science) en su versión 19.0, para determinar diversos cálculos matemáticos y estadísticos con los datos obtenidos ingresados a una base de datos de 
utilidad para la investigación; d) proceso de análisis de los resultados, que consideró usuarios satisfechos a los valores positivos $(+)$ y cero, obtenidos por la diferencia entre las percepciones $(P)$ y las expectativas $(E)$; y usuarios insatisfechos a los valores negativos (-) de la diferencia P-E en la encuesta Servqual.

Se trabajó con un nivel de significancia estadística del 5\%. Se usó la estadística descriptiva a través de: la determinación de proporciones de las variables sociodemográficas y medias de satisfacción de expectativas y percepciones; el cálculo de las diferencias entre expectativas y percepciones por ítems y dimensiones; el nivel de satisfacción según los parámetros anteriormente mencionados; el análisis bivariado de las medias de satisfacción global y de cada una de las dimensiones de la calidad de servicio según variables sociodemográficas, valiéndonos del test $\mathrm{T}$ de Student para evaluar las diferencias entre dos medias, así como la prueba estadística de $\mathrm{Chi}^{2}$ para asociar proporciones con respecto a las variables cualitativas.

El proyecto fue aprobado por el Comité Institucional de tica en Investigación de la Facultad de Medicina de la Universidad San Martín de Porres (Lima, Perú), y el acceso a los consultorios externos del hospital fue autorizado por la dirección del nosocomio. Para mantener el anonimato del sujeto, no se solicitaron nombres ni apellidos u otros datos que pudieran permitir su identificación. La confidencialidad se logró limitando el acceso a los datos solo a los investigadores. A todos los pacientes se les solicitó su consentimiento para incluirlos en el estudio, luego de explicarles sus características y de absolver las preguntas e inquietudes. Este consentimiento se realizó a través de la firma del documento elaborado para tal fin.

\section{Resultados}

En el presente estudio se observó que 52\% (115) de los usuarios eran de sexo femenino, 94.6\% (209) tenían 30 o más años, 85.5\% (189) estaban unidos (as) —casados o convivientes-, 58.4\% (129) eran familiares del beneficiario directo y 72.9\% (161) eran continuadores en la consulta (tabla 1). El tiempo de demora para llegar al establecimiento de salud desde su domicilio fue de 49.8 minutos en promedio, con una mediana de 45 minutos, mientras que el tiempo de espera para la atención tuvo un promedio de 32.5 minutos, con una mediana de 19.8 minutos (tabla 2). 21.3\% de los usuarios manifestó estar satisfecho con la atención recibida, $26.7 \%$ en el componente fiabilidad, $31.2 \%$ en el componente responsabilidad, $48 \%$ en el componente seguridad, $38.9 \%$ en el componente empatía y $32.6 \%$ en el componente tangibles (figura 1 ). 
TABLA 1

Características generales de los usuarios del consultorio externo de gastroenterología del Hospital Central de la Fuerza Aérea del Perú. Mayo, 2014

\begin{tabular}{|l|c|c|}
\hline \multicolumn{1}{|c|}{ Variable } & N & $\%$ \\
\hline Sexo & & \\
\hline Masculino & 106 & 48.0 \\
\hline$\quad$ Femenino & 115 & 52.0 \\
\hline Grupo etario & & \\
\hline 16-29 af́os & 12 & 5.4 \\
\hline 30 a más años & 209 & 94.6 \\
\hline Situación conyugal & & \\
\hline$\quad$ Unido(a) & 189 & 85.5 \\
\hline$\quad$ No unido(a) & 32 & 14.5 \\
\hline Tipo de beneficiario & & \\
\hline$\quad$ Directo & 92 & 41.6 \\
\hline$\quad$ Familiar & 129 & 58.4 \\
\hline Tipo de usuario & & \\
\hline$\quad$ Nuevo & 60 & 27.1 \\
\hline$\quad$ Continuador & 161 & 72.9 \\
\hline
\end{tabular}

Fuente: elaboración propia.

TABLA 2

Tiempo de llegada al establecimiento de salud y tiempo de espera para la atención de los usuarios del consultorio externo de gastroenterología del Hospital Central de la Fuerza Aérea del Perú. Mayo, 2014

\begin{tabular}{|l|c|c|c|}
\hline Variable & Media & E.E. & Mediana \\
\hline Tiempo de llegada al EE.SS. (min) & 49.8 & 2.1 & 45 \\
\hline Tiempo de espera para la atención (min) & 32.5 & 1.3 & 19.8 \\
\hline
\end{tabular}

Fuente: elaboración propia.

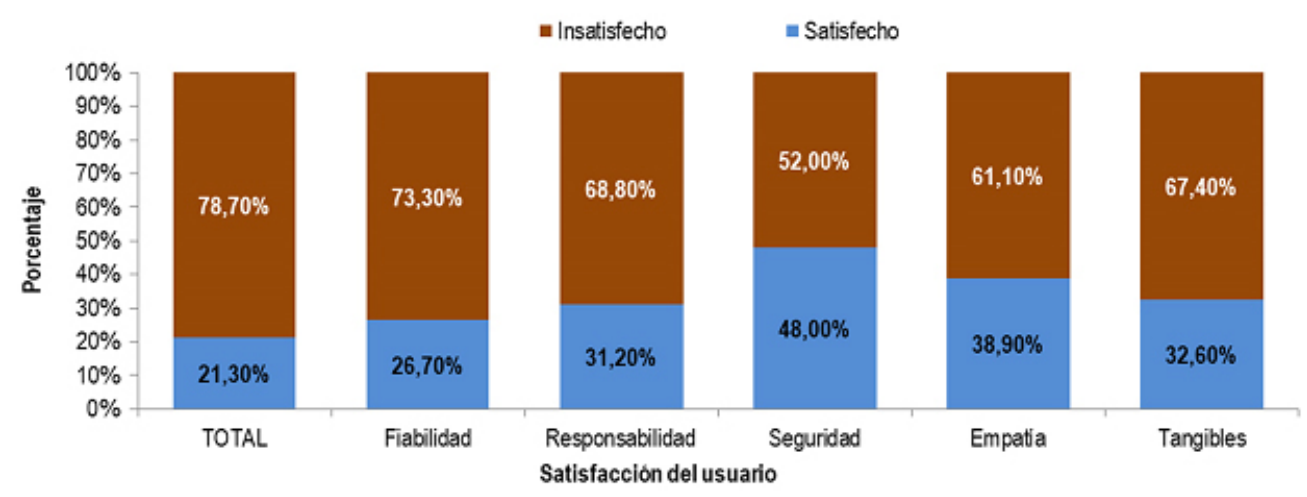

FIGURA 1

Grado de satisfacción de los usuarios del consultorio externo de gastroenterología del Hospital Central de la Fuerza Aérea del Perú. Mayo, 2014 Fuente: elaboración propia.

Del total de encuestados, 75 (70.8\%) de los usuarios de sexo masculino manifestaron estar insatisfechos con la atención recibida. Esta frecuencia fue significativamente inferior $(\mathrm{p}=0.005)$ al número de usuarias de sexo 
femenino que señalaron estar insatisfechas (99; 86.1\%). Asimismo, las usuarias de sexo femenino presentaron puntajes promedio $(-19.97 \pm 1.9)$ significativamente $(\mathrm{p}=0.06)$ inferiores a los obtenidos en los usuarios de sexo masculino $(-13.25 \pm 1.9)$ (tabla 3$)$.

TABLA 3

Grado de satisfacción de los usuarios del consultorio externo de gastroenterología del Hospital Central de la Fuerza Aérea del Perú según características del paciente. Mayo, 2014

\begin{tabular}{|c|c|c|c|c|c|c|c|}
\hline \multirow{3}{*}{$\begin{array}{l}\text { Características } \\
\text { del paciente }\end{array}$} & \multicolumn{4}{|c|}{ Satisfacción de usuario } & \multirow{3}{*}{$\begin{array}{c}\text { Chi cuadrado } \\
\text { (valor de p) }\end{array}$} & \multirow{3}{*}{ Media (E.E.) } & \multirow{3}{*}{$\begin{array}{r}\mathrm{t} \text { de Student } \\
\text { (valor de p) }\end{array}$} \\
\hline & \multicolumn{2}{|c|}{ Satisfecho } & \multicolumn{2}{|c|}{ Insatisfecho } & & & \\
\hline & $\mathrm{n}$ & $\%$ & $\mathrm{n}$ & $\%$ & & & \\
\hline \multicolumn{8}{|l|}{ Sexo } \\
\hline Masculino & 31 & 29.2 & 75 & 70.8 & \multirow{2}{*}{$7.744(0.005)$} & $-13.25(2.0)$ & \multirow{2}{*}{$2.439(0.016)$} \\
\hline Femenino & 16 & 13.9 & 99 & 86.1 & & $-19.97(1.9)$ & \\
\hline \multicolumn{8}{|l|}{ Grupo etario (años) } \\
\hline 16 a 29 & 1 & 8.3 & 11 & 91.7 & \multirow{2}{*}{$1.268(0.260)$} & $-21.17(4.5)$ & \multirow{2}{*}{$-0.761(0.447)$} \\
\hline 30 a más & 46 & 22.0 & 163 & 78.0 & & $-16.49(1.5)$ & \\
\hline \multicolumn{8}{|l|}{ Situación conyugal } \\
\hline Unido & 37 & 19.6 & 152 & 80.4 & \multirow{2}{*}{$2.227(0.136)$} & $-17.10(1.5)$ & \multirow{2}{*}{$-0.625(0.533)$} \\
\hline No unido & 10 & 31.3 & 22 & 68.8 & & $-14.63(4.4)$ & \\
\hline \multicolumn{8}{|c|}{ Tipo de beneficiario } \\
\hline Directo & 27 & 29.3 & 65 & 70.7 & \multirow{2}{*}{$6.147(0.013)$} & $-10.65(1.9)$ & \multirow{2}{*}{$3.807(0.000)$} \\
\hline Familiar & 20 & 15.5 & 109 & 84.5 & & $-21.09(1.9)$ & \\
\hline \multicolumn{8}{|l|}{ Tipo de usuario } \\
\hline Nuevo & 10 & 16.7 & 50 & 83.3 & \multirow{2}{*}{$1.041(0.308)$} & $-21.02(2.6)$ & \multirow{2}{*}{$-1.886(0.061)$} \\
\hline Continuador & 37 & 23.0 & 124 & 77.0 & & $-15.15(1.6)$ & \\
\hline
\end{tabular}

Fuente: elaboración propia.

En relación con el grupo etario, $11(91.7 \%)$ de los usuarios de dieciséis a veintinueve años manifestaron estar insatisfechos con la atención recibida. Esta frecuencia no fue significativamente superior $(\mathrm{p}=0.260)$ al número de usuarios de treinta o más años que señalaron estar insatisfechos (163; 78\%). Asimismo, los usuarios de dieciséis a veintinueve años presentaron puntajes promedio $(-21.17 \pm 4.5)$ inferiores a los obtenidos en los usuarios de treinta a más años $(-16.49 \pm 1.5)$, pero sin significancia estadística $(\mathrm{p}=0.447)$ (tabla 3).

Al determinar la influencia de la situación conyugal, 152 (80.4\%) de los usuarios que manifestaron estar unidos señalaron estar insatisfechos con la atención recibida. Esta frecuencia no fue significativamente superior $(\mathrm{p}=0.136)$ al número de usuarios no unidos que señalaron estar insatisfechos $(22 ; 68.8 \%)$. Además, los usuarios casados y convivientes presentaron puntajes promedio $(-17.10 \pm 1.5)$ inferiores a los obtenidos en los usuarios no unidos $(-14.63 \pm 4.4)$, pero sin significancia estadística $(p=0.533)$ (tabla 3$)$.

Según tipo de beneficiario, observamos que 65 (70.7\%) de los beneficiarios directos señalaron estar insatisfechos con la atención recibida. Esta frecuencia fue significativamente inferior $(\mathrm{p}=0.013)$ al número de familiares de los beneficiarios directos que señalaron estar insatisfechos (109; 84.5\%). Asimismo, los beneficiarios directos presentaron puntajes promedio $(-0.65 \pm 1.9)$ significativamente superiores $(\mathrm{p}=0.000)$ a los obtenidos en los familiares que usan el servicio $(-21.09 \pm 1.9)$ (tabla 3$)$.

En cuanto al tipo de usuario, 50 (83.3\%) de los usuarios nuevos señalaron estar insatisfechos con la atención recibida. Esta frecuencia no fue significativamente superior $(\mathrm{p}=0.308)$ al número de usuarios continuadores que señalaron estar insatisfechos (124; 77.0\%). Asimismo, los usuarios nuevos presentaron 
puntajes promedio $(-21.02 \pm 2.6)$ inferiores a los obtenidos en los usuarios continuadores $(-15.15 \pm 1.6)$, pero sin significancia estadística $(\mathrm{p}=0.061)$ (tabla 3$)$.

También observamos que el tiempo de demora para llegar al establecimiento de salud solo se correlaciona con el componente de responsabilidad, pues a medida que aumenta este tiempo de demora disminuye la satisfacción del usuario $(\mathrm{r}=-0.176 ; \mathrm{p}=0.009)$. En cambio, cuando evaluamos la correlación entre el tiempo de espera para la atención y la satisfacción del usuario, podemos observar que esta última disminuye a medida que aumenta este tiempo de espera $(r=-0.316 ; \mathrm{p}=0.000)$. De igual forma ocurre con todos los componentes: fiabilidad $(\mathrm{r}=-0.383 ; \mathrm{p}=0.000)$; responsabilidad $(\mathrm{r}=-271 ; \mathrm{p}=000)$; seguridad $(\mathrm{r}=-0.175$; $\mathrm{p}=0.000)$; empatía $(\mathrm{r}=-0.266 ; \mathrm{p}=0.000)$ y tangibles $(\mathrm{r}=-0.206 ; \mathrm{p}=0.002)($ tabla 4$)$.

\section{TABLA 4}

Grado de satisfacción según tiempo de llegada al establecimiento de salud y tiempo de espera para la atención de los usuarios del consultorio externo de gastroenterología del Hospital Central de la Fuerza Aérea del Perú. Mayo, 2014

\begin{tabular}{l|c|c|c|c}
\multirow{2}{*}{$\begin{array}{c}\text { Satisfacción } \\
\text { del usuario }\end{array}$} & \multicolumn{2}{|c|}{$\begin{array}{c}\text { Tiempo de llegada al } \\
\text { establecimiento de salud }\end{array}$} & $\begin{array}{c}\text { Tiempo de espera para la } \\
\text { atención }\end{array}$ \\
\cline { 2 - 5 } & $\begin{array}{c}\text { R de } \\
\text { Spearman }\end{array}$ & valor de $\mathrm{p}$ & $\begin{array}{c}\text { R de } \\
\text { Spearman }\end{array}$ & valor de $\mathrm{p}$ \\
\hline Total & $-0,056$ & 0,409 & $-0,316$ & $\mathbf{0 . 0 0 0}$ \\
\hline Fiabilidad & $-0,053$ & 0,43 & $-0,383$ & $\mathbf{0 . 0 0 0}$ \\
\hline $\begin{array}{l}\text { Responsabili } \\
\text { dad }\end{array}$ & -0.176 & $\mathbf{0 . 0 0 9}$ & -0.271 & $\mathbf{0 . 0 0 0}$ \\
\hline Seguridad & 0.044 & 0.516 & -0.175 & $\mathbf{0 . 0 0 0}$ \\
\hline Empatía & 0.200 & 0.763 & -0.266 & $\mathbf{0 . 0 0 0}$ \\
\hline Tangibles & 0.023 & 0.735 & -0.206 & $\mathbf{0 . 0 0 2}$
\end{tabular}

Fuente: elaboración propia.

\section{Conclusión}

Con estos resultados podemos concluir que existe elevado porcentaje de usuarios insatisfechos con el servicio brindado en el consultorio externo de gastroenterología del Hospital Central de la Fuerza Aérea del Perú. Esta insatisfacción se relaciona con el sexo del usuario y el tipo de beneficiario que se atiende en este consultorio, así como con el tiempo de espera para acceder a la atención.

\section{Discusión}

La satisfacción de los pacientes es un componente esencial de la calidad de atención en los servicios de salud. Estos se diseñan y prestan tomando en consideración las expectativas y las preferencias de los usuarios. Por esta razón, la satisfacción del paciente se relaciona tanto con las interacciones clínicas como con aspectos no 
médicos. El Hospital Central de la Fuerza Aérea del Perú y su servicio de gastroenterología no están exentos de esta premisa.

En este estudio podemos observar que la población que acude al consultorio externo de gastroenterología no presenta diferencias significativas con relación al sexo, en su mayoría es adulta o adulta mayor, con unión conyugal - casado o conviviente-. Con mayor frecuencia, son familiares de los beneficiarios directos y continuadores en el servicio. Asimismo, estos usuarios utilizan aproximadamente cincuenta minutos para llegar al establecimiento de salud desde sus domicilios y esperan treinta minutos para ser atendidos en la mitad de los casos estudiados.

En esta población dos de cada diez usuarios del servicio están satisfechos con la atención recibida, resultados diferentes a los encontrados —en otros establecimientos de salud- por Andía et al. [23] y Huiza [24], quienes mencionan mayor proporción de usuarios satisfechos.

Esta insatisfacción se asoció al sexo del usuario, siendo las mujeres quienes presentaron mayor porcentaje y una valoración promedio significativamente inferior a la de los varones; asimismo, los adolescentes y jóvenes tuvieron mayor porcentaje de insatisfacción que los adultos y adultos mayores, además de menores puntajes en la valoración de la satisfacción en relación con la atención recibida, pero estas no fueron estadísticamente significativas. De igual forma ocurrió con la situación conyugal, pues no encontramos relación estadísticamente significativa entre esta variable y la satisfacción del usuario. Similar resultado fue reportado por Casalino-Caripio [8], quien también halló que la insatisfacción global se asociaba al sexo femenino y al grupo etario, siendo los adolescentes los que se mostraban más insatisfechos.

Otro hallazgo de este estudio ha sido la relación de la satisfacción del usuario con el tipo de beneficiario, ya que los familiares muestran mayor porcentaje de insatisfacción que los militares, al igual que menor valoración promedio del servicio recibido. Sin embargo, no se observan diferencias estadísticamente significativas entre los usuarios nuevos y los continuadores.

Adicionalmente, podemos ver que la insatisfacción es mediada, principalmente, por el tiempo de espera que tiene el paciente desde el contacto con la admisión hasta que es atendido, similar al hallazgo reportado por Andía et al. [23]. Si bien el tiempo de demora para llegar desde su domicilio hasta el establecimiento de salud no se correlaciona con la satisfacción global del usuario del servicio, esta sí se correlaciona con el componente de responsabilidad.

Los resultados de este estudio confirman que para brindar atención de manera óptima debe satisfacerse tanto las necesidades médicas como las psicosociales, pues la atención que no satisface las necesidades emocionales o sociales de los pacientes puede dejarlos insatisfechos. De hecho, la garantía de las exigencias de calidad como requisito previo responde a preguntas como: ¿cuáles son los elementos en el proceso de prestación de atención que afectan a la satisfacción del cliente y en qué grado?, ¿en qué medida el significado de calidad difiere entre beneficiarios directos y familiares? y ¿en qué medida la satisfacción del paciente refleja el nivel de la "verdadera" calidad de atención recibida?

Entre las limitaciones del presente estudio podemos señalar que la definición de calidad de atención puede diferir de un país a otro, o al menos de cultura a cultura; nuestro estudio se encuentra dentro del contexto de un hospital militar en nuestro país, por lo que las respuestas de los usuarios podrían estar influidas por las características castrenses del establecimiento; asimismo, los resultados del presente estudio corresponden a una población cautiva del ámbito militar, por lo que deben ser tomados en cuenta para una población de similares características.

\section{Agradecimientos}

Artículo de investigación, basado en el proyecto de investigación Factores asociados al grado de satisfacción del usuario del consultorio externo de gastroenterología del Hospital Central de La Fuerza Aérea del Perú. Mayo, 2014, presentado y sustentado por el Bach. Angello La Torre Mantilla para optar al título de médico cirujano 
en la Facultad de Medicina de la Universidad San Martín de Porres, Lima, Perú. La investigación ha sido financiada por los investigadores.

\section{Referencias}

1. Donabedian A. The Lichfield lecture. Quality assurance in health care: consumers' role. Qual Health Care [serie en internet]. 1992 [acceso: 10 de febrero de 2017]; 1(4):247-51. Disponible en: https://www.ncbi.nlm.nih.gov/p $\mathrm{mc} /$ articles/PMC1055035/

2. Sofaer S, Firminger K. Patient perceptions of the quality of health services. Annu Rev Public Health [serie en internet]. 2005 [acceso: 10 de febrero de 2017]; 26:513-59. Disponible en: http://www.annualreviews.org/doi/full/10.1146/annurev.publhealth.25.050503.153958?url_ver=Z39.88 -2003\&rfr_id=ori\%3Arid\%3Acrossref.org\&rfr_dat=cr_pub\%3Dpubmed\&

3. Gonzáles BI, López P, Marín IA, Haro L, Macías A. Satisfacción de los usuarios de los servicios de salud en el trabajo. Rev Med IMSS [serie en internet]. 2002 [acceso: 10 de febrero de 2017); 40:301-4. Disponible en: http://ww w.medigraphic.com/pdfs/imss/im-2002/im024e.pdf

4. Tarco R, Saco S, Paredes S, Martínez C. Grado de satisfacción laboral del usuario interno en los establecimientos de salud de la red Quispicanchi-Acomayo-Cusco. SITUA [serie en internet]. 2003 [acceso: 10 de febrero de 2017]; (12):36-43. Disponible en: http://sisbib.unmsm.edu.pe/bvrevistas/situa/2004_n23/rsm_grado.htm

5. Traverso-Yépez M, Morais NA. Reinvindicando a subjetividade dos usuarios da Rede Básica de Saùde: para uma humanizacao do atendimento. Cad Saùde Pùblica [serie en internet). 2004 [acceso: 10 de febrero de 2017]; (20):80-8. Disponible en: http://www.scielo.br/scielo.php?script=sci_arttext\&pid=S0102-311X2004000100 022

6. Collins K, O' Cathain A. The continuum of patient satisfaction-from satisfied to very satisfied. Social Sci Med [serie en internet]. 2003 [acceso: 10 de febrero de 2017]; (57): 2465-70. Disponible en: http://www.sciencedirect.co $\mathrm{m} /$ science/article/pii/S0277953603000984

7. Schneider H, Palmer N. Getting to the truth? Researching user views of primary health care. Health Policy Plann [serie en internet]. 2002 [acceso: 10 de febrero de 2017]; (17):32-41. Disponible en: https://www.ncbi.nlm.n ih.gov/pubmed/11861584

8. Casalino-Carpio GE. Calidad de servicio de la consulta externa de Medicina Interna de un hospital general de Lima mediante la encuesta Servqual. Rev Soc Peru Med Interna [serie en internet]. 2008 [acceso: 10 de febrero de 2017]; 21(4):143-52. Disponible en: http://sisbib.unmsm.edu.pe/bvrevistas/spmi/v21n4/pdf/a03v21n4.pdf

9. Villalba R. Calidad en la Atención Primaria de la Salud. Rev Enfermería Global [serie en internet]. 2007 [acceso: 10 de febrero de 2017]; (10):1-15. Disponible en: http://revistas.um.es/eglobal/article/view/199/168

10. Seclén-Palacín J, Darras C. Satisfacción de usuarios de los servicios de salud: Factores sociodemográficos y de accesibilidad asociados. Perú, 2000. An Fac Med [serie en internet]. 2005 [acceso: 10 de febrero de 2017]; 66(2):127-41. Disponible en: http://revistasinvestigacion.unmsm.edu.pe/index.php/anales/article/view/1361 $/ 1156$

11. Baron-Epel O, Dushenat M, Friedman N. Evaluation of the consumer model: relationship between patients' expectations, perceptions and satisfaction with care. Int J Qual Health Care [serie en internet]. 2001 [acceso: 10 de febrero de 2017]; 13(4):317-23. Disponible en: https://www.ncbi.nlm.nih.gov/pubmed/11560351

12. Baltussen R, Yê Y, Hadad S, Sauerborn R. Perceived quality of care of primary care services in Burkina Faso. Health Policy and Plann [serie en internet]. 2002 [acceso: 10 de febrero de 2017]; 17(1):42-8. Disponible en: https:// www.ncbi.nlm.nih.gov/pubmed/11861585

13. Gross R, Tabenkin H, Porta A, Heymann A, Greenstein M, Porter B, Matzliach R. The relationship between primary care physicians' adherence to guidelines for the treatment of diabetes and patient satisfaction: findings from a pilot study. Fam Pract [serie en internet]. 2003 [acceso: 10 de febrero de 2017]; 20(5):563-9. Disponible en: https://www.ncbi.nlm.nih.gov/pubmed/14507799 
14. Miyahira J. Calidad en los servicios de salud ¿Es posible? Rev. Med. Hered [serie en internet]. 2001 [acceso: 10 de febrero de 2017]; 12(3):75-7. Disponible en: http://www.scielo.org.pe/scielo.php?script=sci_arttext\&pid=S1 018-130X2001000300001

15. Wong LL, Ooi SBS, Gogh LG. Patients' complaints in a hospital emergency department in Singapore. Singapore Med J [serie en internet]. 2007 [acceso: 10 de febrero de 2017]; 48(11):990-5. Disponible en: http://smj.sma. org.sg/4811/4811a2.pdf

16. Giugliani C, Gault N, Fares V, Jegu J, Eleni dit Trolli S, Biga J, Vidal-Trecan G. Evolution of patients' complaints in a French university hospital: is there a contribution of a law regarding patients' rights? BMC Health Serv Res [serie en internet]. 2009 [acceso: 10 de febrero de 2017]; (9):141. Disponible en: http://bmchealthservres.bio medcentral.com/articles/10.1186/1472-6963-9-141

17. Nketiah-Amponsah E, Hiemenz U. Determinants of consumer satisfaction of health care in Ghana: does choice of health care provider matter? Global Journal of Health Science [serie en internet]. 2009 [acceso: 10 de febrero de 2017]; 1(2):50-61. Disponible en: http://www.ccsenet.org/journal/index.php/gjhs/article/view/2597/3473

18. Shimabuku R, Huicho L, Fernández D, Nakachi G, Maldonado R, Barrientos A. Niveles de insatisfacción del usuario externo en el Instituto Nacional de Salud del Niño de Lima, Perú. Rev Peru Med Exp Salud Publica [serie en internet]. 2012 [acceso: 10 de febrero de 2017]; 29(4):483-9. Disponible en: http://www.scielo.org.pe/pdf /rins/v29n4/a10v29n4.pdf

19. Nebot C, et al. La opinión de los usuarios como oportunidad de mejora en atención primaria. Rev Aten Primaria [serie en internet]. 2005 [acceso: 10 de febrero de 2017]; 35(6):290-4. Disponible en: http://www.elsevier.es/e s-revista-atencion-primaria-27-articulo-la-opinion-los-usuarios-como-13073414

20. Bara AC, van den Heuvel WJA, Maarse JAM, van Dijk JP. Users' satisfaction with the Romanian health care system: and evaluation of recent health care reforms. European Journal of Public Health [serie en internet]. 2002 [acceso: 10 de febrero de 2017]; 12(4):39-40. Disponible en: http://www.rug.nl/research/portal/files/2994140/c5.pdf

21. Ministerio de Salud del Perú, Proyecto 2000. Calidad del servicio de salud desde la percepción de la usuaria: Diagnóstico de necesidades de comunicación [monografía en internet]. Lima: Pathfinder International, USAID; 2002 [acceso: 10 de febrero de 2017]. Disponible en: http://bvs.minsa.gob.pe/local/P2000/664_MS-P-20002 76.pdf

22. Jackson J, Chamberlin J, Kroenke K. Predictors of patient satisfaction. Soc Sci Med [serie en internet]. 2001 [acceso: 10 de febrero de 2017]; 52: 609-20. Disponible en: http://www.sciencedirect.com/science/article/pii/S02779 53600001647

23. Andía CM, Pineda AM, Sottec V, Santos JR, Molina ME, Romero Z. Satisfacción del usuario de los servicios de consulta externa del hospital I Espinar, abril 2002. SITUA [serie en internet]. 2002 [acceso: 10 de febrero de 2017]; 20:18-22. Disponible en: http://sisbib.unmsm.edu.pe/bvrevistas/situa/2002_n20/pdf/satisfac_usuario sconsulta.pdf

24. Huiza GA. Satisfacción del usuario externo sobre la calidad de atención de salud en el Hospital de la Base Naval. Callao. Octubre-diciembre 2003 [monografía en internet]. Lima: Universidad Nacional Mayor de San Marcos; 2006 [acceso: 10 de febrero de 2017]. Disponible en: http://cybertesis.unmsm.edu.pe/bitstream/cybertesis/18 91/1/Huiza_gg.pdf

25. Niño BS, Perales JCT, Chavesta XP, Leguía JA, Díaz C. Calidad de servicio en la consulta externa de un hospital de la región Lambayeque octubre 2010. Rev Cuerpo Méd HNAAA [serie en internet]. 2012 [acceso: 10 de febrero de 2017]; 5(1):5-9. Disponible en: https://dialnet.unirioja.es/servlet/articulo?codigo $=4061256$

26. Cabrera-Arana GA, Bello-Parías LD, Londoño-Pimienta JL. Calidad percibida por usuarios de hospitales del Programa de Reestructuración de Redes de Servicios de Salud de Colombia. Rev Salud Pública [serie en internet]. 2008 [acceso: 10 de febrero de 2017]; 10(4):593-604. Disponible en: http://www.scielosp.org/pdf/rsap/v10n 4/v10n4a09.pdf

27. Chávez P, Ramos W, Galarza C. Nivel de satisfacción del usuario en los consultorios externos de dermatología del Hospital Nacional Dos de Mayo. Diciembre 2006. Dermatol Perú [serie en internet]. 2009 [acceso: 10 de febrero de 2017]; 19(1):22-31. Disponible en: http://sisbib.unmsm.edu.pe/BVRevistas/dermatologia/v19_n1/pdf/a0 $4 \mathrm{v} 19 \mathrm{n} 1 . \mathrm{pdf}$ 
28. Ortiz RM, Muñoz S, Torres E. Satisfacción de los usuarios de 15 hospitales de Hidalgo, México. Rev Esp Salud Pública [serie en internet]. 2004 [acceso: 10 de febrero de 2017]; (78):527-37. Disponible en: http://scielo.isc iii.es/pdf/resp/v78n4/original5.pdf

29. Castro MR, Villagarcia H, Saco S. Satisfacción del usuario de los servicios de hospitalización del Hospital Antonio Lorena: mayo-agosto del 2003. SITUA. 2004 [acceso: 10 de febrero de 2017]; (23):47-53. Disponible en: http ://sisbib.unmsm.edu.pe/bvrevistas/situa/2004_n23/enPDF/a09.pdf

30. Guía técnica para la evaluación de la satisfacción del usuario externo en los establecimientos de salud y servicios médicos de apoyo [monografía en internet]. Lima: Ministerio de Salud del Perú; 2011 [acceso: 15 de enero de 2014]. Disponible en: http://bvs.minsa.gob.pe/local/minsa/2252.pdf

31. Cabello E, Chirinos JL. Validación y aplicabilidad de encuestas Servqual modificadas para medir la satisfacción de usuarios externos en servicios de salud. Rev Med Hered [serie en internet]. 2012 [acceso: 15 de enero de 2014]; 23(2):88-95. http://www.upch.edu.pe/vrinve/dugic/revistas/index.php/RMH/article/viewFile/1037/1003

\section{Notas}

* Artículo de investigación

\section{Licencia Creative Commons CC BY 4.0}

Como citar este artículo: La Torre Mantilla AR, Oyola García AE, Quispe Ilanzo MP. Factores asociados al grado de satisfacción del usuario del consultorio externo de gastroenterología del Hospital Central de la Fuerza Aérea del Perú. Mayo, 2014. Rev Gerenc Polít Salud. 2017; 17(34): 1-11. https://doi.org/10.1114 4/Javeriana.rgps17-34.fasu 